\title{
WILEY-VCH
}

DOI: 10.1002/((please add manuscript number))

Article type: Communication

\section{Mesoporous Silica Supraparticles for Sustained Inner-Ear Drug Delivery}

Yajun Wang, Andrew K. Wise, Justin Tan, James W. Maina, Robert K. Shepherd, and Frank Caruso*

Prof. Y. Wang, ${ }^{[+]}$Mr. J. W. Maina, Prof. F. Caruso

Department of Chemical and Biomolecular Engineering

The University of Melbourne

Parkville, Victoria 3010, Australia

E-mail: fcaruso@unimelb.edu.au

Dr. A. K. Wise, ${ }^{[+]}$Dr. J. Tan, Prof. R. K. Shepherd

Bionics Institute

East Melbourne, Victoria 3002, Australia

Department of Otolaryngology, The University of Melbourne

Dr. A. K. Wise, ${ }^{[+]}$Dr. J. Tan , Prof. R. K. Shepherd

Department of Otolaryngology

The University of Melbourne

East Melbourne, Victoria 3002, Australia

Prof. Y. Wang

Department of Chemistry

Fudan University

Shanghai, 200433, P. R. China

Keywords: mesoporous materials, supraparticles, drug delivery, neurotrophic factors, sensorineural hearing loss 


\section{WILEY-VCH}

Nanotechnology offers new possibilities for delivering drugs to combat various diseases. ${ }^{[1]}$ Hearing loss is a major health problem and the treatment of hearing-related diseases requires efficient transfer of therapeutics to the target organ in the inner ear - the cochlea. ${ }^{[2]}$ Approaches using systemic drug delivery to treat hearing-related diseases have achieved limited success due to difficulties accessing the inner. ${ }^{[3]}$ Local administration of drugs via absorption from the middle ear has been widely studied. ${ }^{[3]}$ However, clearance of substances by ciliated epithelia in the tympanic cavity and uncontrolled diffusion away from the inner ear hampers efficient uptake. ${ }^{[3]}$ Recently, we reported that surgical implantation of neurotrophinloaded nanoporous polymer particles in the cochlea improved the survival rate of primary auditory neurons (ANs). ${ }^{[4]}$ These particles have small diameters up to ca. $3 \mu \mathrm{m}$, so surgical grade gel foam was used to retain these particles within the inner ear. Whereas this approach reduces outflow of the particles from the cochlea, the amount of neurotrophins which can be encapsulated is limited by the capacity of the delivery system and the narrow space within the cochlea. Furthermore, the small-sized particles may inadvertently disperse into the cerebral spinal fluid via the cochlear aqueduct following cochlear surgery. ${ }^{[5]}$ For a more efficient and easier-to-handle drug-delivery system applied in the inner ear, particles with significantly larger sizes and superior loading capacity are desirable.

In designing carriers for more effective drug delivery to the inner ear, we report the first preparation of a new class of drug carriers based on mesoporous silica (MS) building blocks, termed MS supraparticles (MS-SPs), and use these to deliver neurotrophins in order to rescue neuronal cells that have lost their endogenous neurotrophin supply. The strategy for the assembly of MS-SPs with a well-controlled number of nanoparticles involves trapping a given number of particles together in a confined space and allowing capillary forces to form the supraparticle clusters. ${ }^{[6]}$ In the past decade, MS particle-based therapeutic carriers have attracted considerable interest because MS-engineered particles can provide a number of 


\section{WILEY-VCH}

advantages in drug delivery, such as high surface area, facile surface chemistry modification, adjustable pore sizes, tunable particle sizes, excellent biocompatibility, as well as the ability to protect the drug from endogenous proteases that cause degradation of the drug molecule. ${ }^{[7]}$ Hence, MS particles have been widely used to encapsulate various compounds and for the template synthesis of diverse nanostructured materials for drug delivery applications. ${ }^{[8]}$ Research in the biomedical applications of MS materials has mainly focused on systemic drug delivery applications, in which small-sized particles with diameters ranging from tens of nanometers to around $500 \mathrm{~nm}$ are frequently used. ${ }^{[7 \mathrm{~b}]}$ The prepared MS-SPs reported herein possess large pore volumes and high surface areas, allowing the loading of large amounts of drug, thus providing therapeutically relevant concentrations for local treatment.

The MS-SPs are synthesized via self-assembly of MS nanoparticles in confined droplets under the action of capillary forces (Scheme 1). MS nanoparticles (diameter, ca. $400 \mathrm{~nm}$ ) with a bimodal pore structure ( 2-3 $\mathrm{nm}$ and $15-30 \mathrm{~nm}$ ) were used as the building blocks in this study. ${ }^{[9]}$ The bigger mesopores $(15-30 \mathrm{~nm})$ are useful for the transport of biomacromolecules (e.g., protein drugs) through MS particles. ${ }^{[7 b]}$ The MS particles were pre-dispersed in water to form stable colloids with a particle concentration of $5 \mathrm{wt} \%$. Droplets of MS nanoparticles with a volume of 0.5 to $2.0 \mu \mathrm{L}$ were then applied to a paraffin film-covered surface and the drops were dried under air flow. The size of the MS-SPs assembled is governed by the volume of the colloidal suspensions applied in the droplet. The droplets retained a nearspherical shape on the hydrophobic surface of paraffin film, observed by the high contact angle of water on the paraffin film. Air flow was necessary to accelerate the evaporation of water from the droplets. The free water volume inside the droplets reduces during the drying process (ca. $10 \mathrm{~min}$ ), thus forcing the MS nanoparticles to self-assemble into a closely packed structure under capillary force action. Without air flow, significantly longer times (ca. 1-3 h, depending on the droplet size) are required to dry the droplets, and disk- to doughnut-shaped assemblies were obtained after water evaporation. This is likely caused by sedimentation of 


\section{WILEY-VCH}

the MS nanoparticles on the paraffin film surface during the relatively long drying period. The air-dried supraparticles were then removed from the paraffin film and annealed at $650{ }^{\circ} \mathrm{C}$ in a ceramic container. The calcination process can condense the Si-OH groups on the surface of the siliceous nanoparticles to form a Si-O-Si linkage between the MS nanoparticles, ${ }^{[10]}$ which enhances the mechanical stability of the assembled MS nanoparticles. It is noted that supraparticles without the heat treatment step are prone to cracking when they are transferred into aqueous solutions. The calcined MS-SPs maintained a near-spherical shape, and those assembled with $0.5,1.0$ and $2.0 \mu \mathrm{L}$ of MS nanoparticles have a diameter of ca. 580, 710 and $850 \mu \mathrm{m}$, respectively (Figure 1a-c).

The inner structure of the MS-SPs was examined by transmission electron microscopy (TEM) of ultramicrotomed samples with a slice thickness of ca. $90 \mathrm{~nm}$ (Figure 1d-f). The distribution of the MS nanoparticles throughout the MS-SPs can be seen in Figure 1d. At higher magnification, the porosity of the MS-SPs is apparent, with pores distributed across three length scales (Figure 1f). The macropores with a pore size of ca. 100-200 nm are formed through the close packing of the primary MS nanoparticles. The larger mesopores between $15-30 \mathrm{~nm}$ and the smaller mesopores in the 2-3 $\mathrm{nm}$ range originate from the building blocks of the MS nanoparticles. The interconnected macroporous channels are useful to transfer biomacromolecules throughout the supraparticles, while the abundant, well-accessible mesopores can provide a high surface area for loading various drugs with a broad range of sizes (i.e., small drugs, proteins and nucleic acids).

Nitrogen sorption isotherms of the MS-SPs (as shown in Figure 1a) exhibited type IV isotherms with two distinct adsorption steps at the relative pressure of $0.30-0.60$ and $0.80-0.95$, respectively (Figure 2a). MS-SPs have a surface area of $888 \mathrm{~m}^{2} \mathrm{~g}^{-1}$ and a pore volume of 2.0 $\mathrm{cm}^{3} \mathrm{~g}^{-1}$. The pore distribution curve (Figure 2a, inset) indicates that the MS-SPs have a bimodal mesopore structure, that is, smaller pores in the 2-3 nm range, and the larger pores ranging from 15 to $30 \mathrm{~nm}$. 


\section{WILEY-VCH}

The capacity of the MS-SPs as hosts for the loading of therapeutic proteins was investigated using brain-derived neurotrophic factor (BDNF). Like other neurotrophins, BDNF is a basic protein $\left(M_{\mathrm{W}} 13 \mathrm{kDa}\right)$ with an isoelectronic point of $\mathrm{pH} 10$ and has a profound action on neuronal rescue. ${ }^{[11]}$ We conducted adsorption studies, where six MS-SPs (ca. 580 $\mu \mathrm{m}$ in diameter) were soaked in $100 \mu \mathrm{L}$ of $0.1 \mathrm{mg} \mathrm{mL}^{-1}$ BDNF solution in water. The efficient loading of BDNF in the MS-SPs was indicated by the dramatic decrease of the BDNF initial concentration from $0.10 \mathrm{mg} \mathrm{mL}^{-1}$ to ca. $0.02 \mathrm{mg} \mathrm{mL}^{-1}$ after three days of incubation at room temperature. The amount of encapsulated BDNF in each supraparticle was ca. $1.33 \mu \mathrm{g}$, corresponding to a BDNF local concentration of ca. $12 \mathrm{mg} \mathrm{mL}^{-1}$ in the MS-SPs, which is two orders of magnitude higher than the BDNF concentration in the original incubating solution. This efficient loading can be attributed to the high porosity of the MS-SPs and the strong electrostatic interactions between the residual amine groups on the protein and the abundant Si-OH groups on the nanoporous siliceous materials.

The in vitro release profiles of the BDNF were investigated by incubating three BDNFloaded MS-SPs in phosphate-buffered saline (PBS) at $37^{\circ} \mathrm{C}$. The amount of BDNF released was monitored by an enzyme-linked immunosorbent assay (ELISA). Minimal leakage of BDNF (ca. 2 ng) from the MS-SPs was found during storage in water at $4{ }^{\circ} \mathrm{C}$, indicating a strong affinity between the BDNF protein and the MS-SPs. It is also noted that the slower diffusion rate of protein at low temperature $\left(4^{\circ} \mathrm{C}\right)$ in particular, inside the nanoporous channels of MS-SPs with a large particle size, could also contribute to the negligible leakage of the protein during storage. The cumulative release of BDNF over a period of 70 days is depicted in Figure 2b. A robust release within the first 28 days was observed, followed by a more gradual release to the end of the incubation period. In total, approximately $10 \%$ of the loaded BDNF was monitored to be released, suggesting a strong affinity between BDNF and the MS-SPs. BDNF release is likely governed by its diffusion through the channels of the MS-SPs. The driving force is a concentration gradient of the BDNF between the MS-SPs and 


\section{WILEY-VCH}

the surrounding PBS medium. Gradual degradation of the MS-SPs (to soluble silicic acid species) in PBS under physiological conditions also contributes to elution of the BDNF. ${ }^{\text {[7a] }}$ Previous studies on the clearance of silica particles in the body showed that the silica was excreted in the urine in the form of silicic acid or organic silica species. ${ }^{[12]}$ MS-SPs were observed to degrade, as indicated by the decrease in the density of MS-SPs after long-term (one month) incubation in PBS. The white MS-SPs gradually turned semi-transparent, accompanied by a ca. 50\% decrease in diameter after 70 days of incubation, suggesting partial dissolution of the MS-SPs.

To determine the surgical approach and suitability of using MS-SPs for sustained delivery of BDNF to the cochlea, a pilot experiment was carried out in a guinea pig pre-deafened using procedures previously described. ${ }^{[4]} \mathrm{A}$ small cochleostomy was drilled in the basal turn of the cochlea and seven BDNF-loaded MS-SPs were implanted inside the cochlea. The surgical procedure was repeated for the contralateral side, except that unloaded MS-SPs were used for the implant. After 28 days of treatment the guinea pig was sacrificed. Cochleae were dissected, fixed, cryo-sectioned (at a thickness of $12 \mu \mathrm{m}$ ) and then stained. Representative histological images of the cochlea implanted with MS-SPs containing BDNF and the contralateral cochlea implanted with control MS-SPs are shown in Figure 3.

The outcomes of this experiment indicate that the MS-SPs are suitable for implantation into the cochlea and provide evidence that BDNF can be released at therapeutic levels required for nerve survival. Greater survival of ANs (arrowhead) was observed in the BDNFloaded MS-SPs treated cochlea (Figure 3a) compared with the contralateral cochlea treated with the MS-SPs alone (Figure 3b). The density of the remaining ANs in cochlear sections shown in Figure 3 was determined by counting the auditory neurons and measuring the area of spiral canal of cochlea (Rosenthal's canal). The AN density in the ear treated with BDNFloaded MS-SPs was $1302 \mathrm{AN} \mathrm{mm}^{-2}$ (Figure 3a), 2.7 times higher than the auditory neuron survival in the control ear $\left(484 \mathrm{AN} \mathrm{mm}^{-2}\right)$. 


\section{WILEY-VCH}

In conclusion, we have demonstrated that MS-SPs can be synthesized via self-assembly of MS nanoparticles under capillary force action in confined droplets applied on a hydrophobic surface. Robust MS-SPs were obtained after heat treatment of the as-assembled MS-SPs. TEM data show that the MS-SPs have hierarchical porous structures with pore sizes distributed across three levels: 100-200 nm, 15-30 nm, and 2-3 nm. The prepared MS-SPs were used as effective carriers for therapeutically relevant concentrations of BDNF. In vitro release experiments showed that the loaded BDNF in the MS-SPs was released from the supraparticles over a period of 70 days, with a relatively quicker release rate in the first month. A pilot animal study demonstrated that MS-SPs were suitable for chronic intracochlear implantation and that the BDNF released from the MS-SPs was biologically active and could efficiently rescue primary auditory neurons in a sensorineural hearing loss model. Further, the concept of using MS-SPs as local drug delivery vehicles should be easily transferable to other drugs for other treatments, including antibiotics for reducing surgical symptoms, and in particular to reduce the effects of surgical trauma and preserve residual hearing and AN population in the field of cochlear implants.

\section{Experimental Section}

Synthesis of MS Supraparticles: The primary building blocks, MS nanoparticles, were prepared according to a literature method. ${ }^{[9]}$ The MS nanoparticles were dispersed in Milli-Q water with a particle concentration of $5 \mathrm{wt} \%$ and briefly sonicated to form a stable colloidal suspension. A 0.5 to $2.0 \mu \mathrm{L}$ aliquot of the MS nanoparticle dispersion was then applied to a flat surface, which was pre-covered with a paraffin film. The droplets were dried under air flow to drive assembly of the MS nanoparticles into MS-SPs via capillary force action. The size of the MS-SPs can be controlled by the volume of the nanoparticle dispersion applied in the droplet. The air-dried MS-SPs were then removed from the paraffin film and transferred into a ceramic container and further dried in a furnace at $120^{\circ} \mathrm{C}$ overnight. Following this, the 


\section{WILEY-VCH}

furnace temperature was slowly increased to $650{ }^{\circ} \mathrm{C}$ (in $12 \mathrm{~h}$ ) and held at this temperature for $6 \mathrm{~h}$. After the furnace cooled down to room temperature, the MS-SPs were collected in a plastic centrifuge tube for further use.

BDNF Loading: Six MS-SPs (as shown in Figure 1a) were sterilized via soaking the MS-SPs in $100 \mu \mathrm{L}$ of ethanol $(80 \mathrm{v} / \mathrm{v} \%)$ for $4 \mathrm{~h}$. After exchanging the ethanol through rinsing with 100 $\mu \mathrm{L}$ of Milli-Q water six times, the MS-SPs were left in $90 \mu \mathrm{L}$ of Milli-Q water. $10 \mu \mathrm{g}$ of BDNF (1 mg mL ${ }^{-1}$ in Milli-Q water) was then added to the sterilized MS-SPs and incubated at ambient temperature for 3 days with occasional hand shaking. The BDNF-loaded MS-SPs were stored at $4{ }^{\circ} \mathrm{C}$ and briefly rinsed once with $20 \mu \mathrm{L}$ of Milli-Q water before use in surgery. In vitro BDNF Release: Three MS-SPs were incubated in $100 \mu \mathrm{L}$ of phosphate buffered saline (PBS, pH 7.3) at $37^{\circ} \mathrm{C}$ in a paraffin film sealed $1.5 \mathrm{~mL}$ centrifugation tube. At various time intervals, $90 \mu \mathrm{L}$ of solution was removed for BDNF immunoassay experiments and $90 \mu \mathrm{L}$ of fresh PBS was added to continue the release process. BDNF measurements were performed using a BDNF Emax ImmunoAssay (Promega) and following the manufacturer's protocol. Animal Surgery: The St Vincent's Hospital Melbourne Animal Ethics Committee approved the use of the Dunkin-Hartley (strain) guinea pig used in this study. The guinea pig was deafened with frusemide (130 mg per kg of body weight, delivered intravenously) and kanamycin (420 mg per kg of body weight, delivered subcutaneously) using procedures previously described. ${ }^{[13]}$ Hearing thresholds were determined under anaesthesia by recording the auditory brainstem response elicited from a click stimulus, as described elsewhere. ${ }^{[4]}$ Surgery was performed under aseptic conditions one week after deafening. The guinea pig was anaesthetized with an intramuscular injection of ketamine (60 mg per kg body weight) and xylazine (4 mg per kg body weight). An incision was performed above the external ear to expose the bulla and a hole was drilled through the bulla to expose the basal turn of the cochlea. A small cochleostomy was drilled in the basal turn of the cochlea and traces of exuding perilymphatic fluids were drained using light suction. A total of seven MS-SPs were 


\section{WILEY-VCH}

implanted. The surgical procedure was repeated for the contralateral side. One ear received control (unloaded) MS-SPs and the other ear received MS-SPs that contained BDNF. Finally, muscle tissues were used to plug the cochleostomy and the incision was closed with two layers of sutures.

Histology: After 28 days of treatment the guinea pig was sacrificed with a lethal dose of sodium pentobarbitone and perfused intracardially with a warm physiological saline solution $(0.9 \% \mathrm{wt} / \mathrm{vol})$ containing $0.1 \%(\mathrm{vol} / \mathrm{vol})$ heparin sodium and $0.025 \%$ (wt/vol) sodium nitrate, followed by a solution of $10 \%$ Neutral Buffered Formalin $\left(\mathrm{pH} 7.4,4{ }^{\circ} \mathrm{C}\right)$. Cochleae were rapidly dissected and the middle ear ossicles removed to allow penetration of the fixative. Cochleae were fixed for $1 \mathrm{~h}$ at room temperature with shaking. Following fixation, they were decalcified in 10\% ethylene diamine tetra-acetic acid dissolved in PBS (pH 7.4) to soften the tissues for sectioning. After decalcification, they were incubated in 30\% sucrose dissolved in PBS before overnight infiltration and final embedding in Optimum Cutting Temperature compound (Sakura, Tokyo, Japan) as described previously. ${ }^{[14]}$ Cochleae were cryo-sectioned along the plane of the vertical, central axis at a thickness of $12 \mu \mathrm{m}$ and stained with haematoxylin and eosin.

\section{Acknowledgements}

This work was supported by the National Health and Medical Research Council (NHMRC) Project Grant APP1005071 (R.K.S., F.C., J.T., Y.W., A.K.W) and Project Grant APP1064375 (A.K.W., F.C. and R.K.S.), by the Australian Research Council under the Australian Laureate Fellowship scheme (F.C., 120100030) and the National Institutes of Health (USA) HHS-N263-2007-00053-C (R.K.S.). The Bionics Institute acknowledges the support it receives from the Victorian government through its Operational Infrastructure Support Program. Y.W. acknowledges support from The National Young 1000 Talents Program of China and the National Natural Science Foundation of China (21373059). Y. Wang and A. K. Wise contributed equally to this work.

Received: ((will be filled in by the editorial staff))

Revised: ((will be filled in by the editorial staff)) Published online: ((will be filled in by the editorial staff)) 


\section{WILEY-VCH}

[1] a) O. C. Farokhzad, R. Langer, Adv. Drug Deliv. Rev. 2006, 58, 1456-1459; b) D. F.

Emerich, C. G. Thanos, J. Drug Target. 2007, 15, 163-183; c) L. Zhang, F. X. Gu, J. M. Chan, A. Z. Wang, R. S. Langer, O. C. Farokhzad, Clin. Pharmacol. Ther. 2008, 83, 761-769; d) K. Riehemann, S. W. Schneider, T. A. Luger, B. Godin, M. Ferrari, H. Fuchs, Angew. Chem. Int. Ed. 2009, 48, 872-897; e) Y. Wang, H. Lomas, L. Hosta-Rigau, F. Caruso, Phys. Chem. Chem. Phys. 2011, 13, 4782-4801.

[2] a) T. G. Landry, A. K. Wise, J. B. Fallon, R. K. Shepherd, Hearing Res. 2011, 282, 303-313; b) A. K. Wise, L. N. Gillespie, J. Neural. Eng. 2012, 9, 065002.

[3] C. O. Pritz, J. Dudás, H. Rask-Andersen, A. Schrott-Fischer, R. Glueckert, Nanomedicine 2013, 8, 1155-1172.

[4] J. Tan, Y. Wang, X.P. Yip, F. Glynn, R.K. Shepherd, F. Caruso, Adv. Mater. 2012, 24, 3362-3366.

[5] a) T. Rivera, L. Sanz, G. Camarero, I. Varela-Nieto, Curr. Drug Deliv. 2012, 9, 231-

242. b) B. Coleman, J. Hardman, M. de Silva, S. Epp, A. Coco, J. Crook, R. K. Shepherd, J. Cell Transplantation 2006, 15, 369-380.

[6] V. Rastogi, S. Melle, O. G. Calderón, A. A. Garciá, M. Marquez, O. D. Velev, Adv. Mater. 2008, 20, 4263-4268.

[7] a) N. Ehlert, P. P. Mueller, M. Stieve, T. Lenarz, P. Behrens, Chem. Soc. Rev. 2013, 42, 3847-3861; b) Y. J. Wang, A. D. Price, F. Caruso, J. Mater. Chem. 2009, 19, 6451-6464. [8] a) Y. Wang, A. M. Yu, F. Caruso, Angew. Chem. Int. Ed. 2005, 44, 2888-2892; b) Y. Wang, F. Caruso, Adv. Mater. 2006, 18, 795-800; c) Y. Wang, A. S. Angelatos, D. E. Dunstan, F. Caruso, Macromolecules 2007, 40, 7594-7600; d) Y. Wang, V. Bansal, A. N. Zelikin, F. Caruso, Nano Lett. 2008, 8, 1741-1745; e) Y. Wang, Y. Yan, J. W. Cui, L. Hosta-Rigau, J. K. Heath, E. C. Nice, F. Caruso, Adv. Mater. 2010, 22, 4293-4297; f) D. Mertz, P. Tan, Y. Wang, T. K. Goh, A. Blencowe, F. Caruso, Adv. Mater.2011, 23, 5668-5673. 


\section{WILEY-VCH}

[9] J. G. Wang, H. J. Zhou, P. C. Sun, D. T. Ding, T. H. Chen, Chem. Mater. 2010, 22, 3829-3831.

[10] Y. Wang, F. Caruso, Adv. Funct. Mater. 2004, 14, 1012-1018.

[11] a) A. K. McAllister, L. C. Katz, D. C. Lo, Annu. Rev. Neurosci. 1999, 22, 295-318; b)

L. Minichiello, M. Korte, D. Wolfer, R. Kuhn, K. Unsicker, V. Cestari, C. Rossi-Arnaud, H. P. Lipp, T. Bonhoeffer, R. Klein, Neuron 1999, 24, 401-414; c) J. R. Chan, J. M. Cosgaya, Y. J. Wu, E. M. Shooter, Proc. Natl. Acad. Sci. U.S.A. 2001, 98, 14661-14668.

[12] Q. J. He, Z. W. Zhang, F. Gao, Y. P. Li, J. L. Shi, Small, 2011, 7, 271-280.

[13] A. K. Wise, R. Richardson, J. Hardman, G. Clark, S. O'Leary, J. Comp. Neurol. 2005, 487, 147-165.

[14] A. K. Wise, C. R. Hume, B. O. Flynn, Y. S. Jeelall, C. L. Suhr, B. E. Sgro, S. J. O'Leary, R. K. Shepherd, R. T. Richardson, Mol. Ther. 2010, 18, 1111-1122. 


\section{WILEY-VCH}
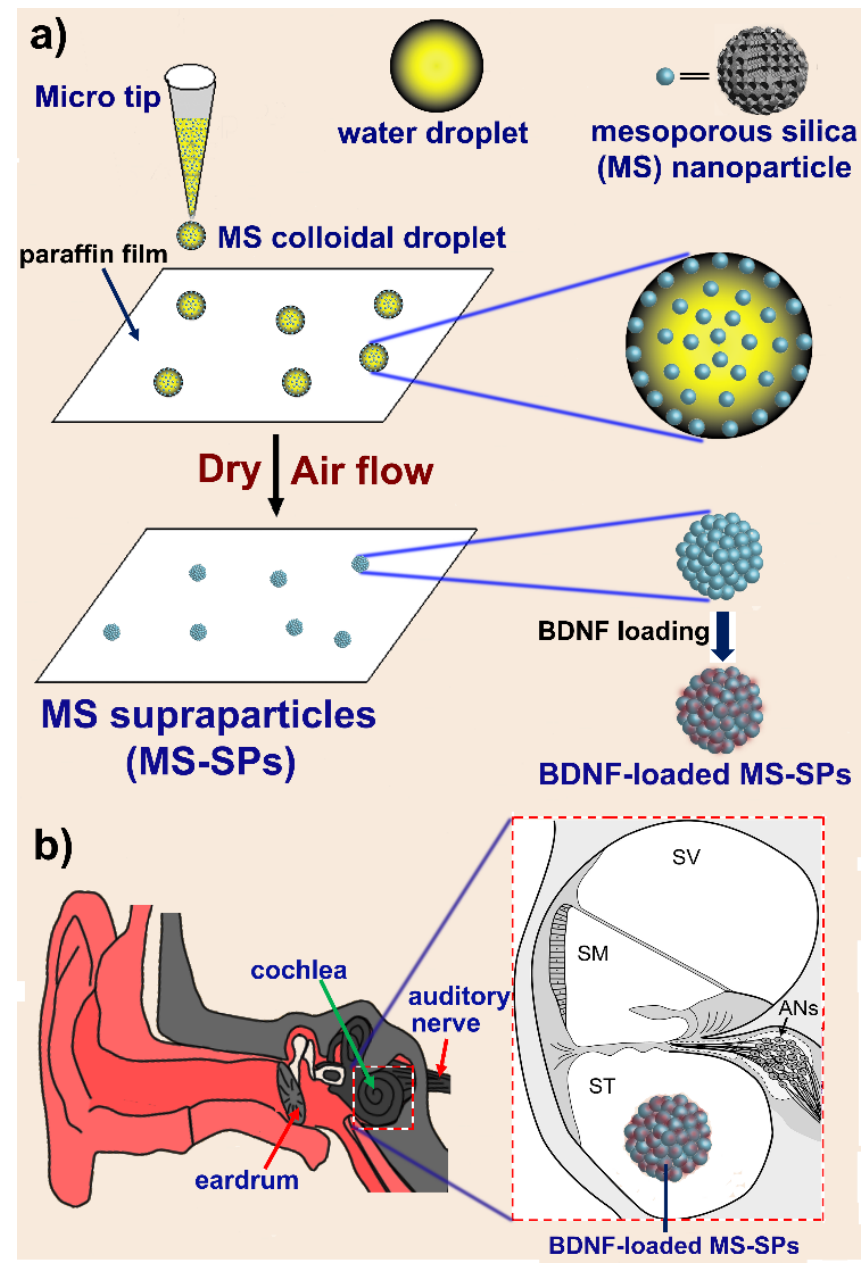

Scheme 1. Schematic representation of the preparation of MS-SPs (a) and surgical implanting of MS-SPs in the inner ear (b). BDNF: brain-derived neurotrophic factor; ST: Scala Tympani; SV: Scala Vestibuli; SM: Scala Media; AN: Primary Auditory Neurons.

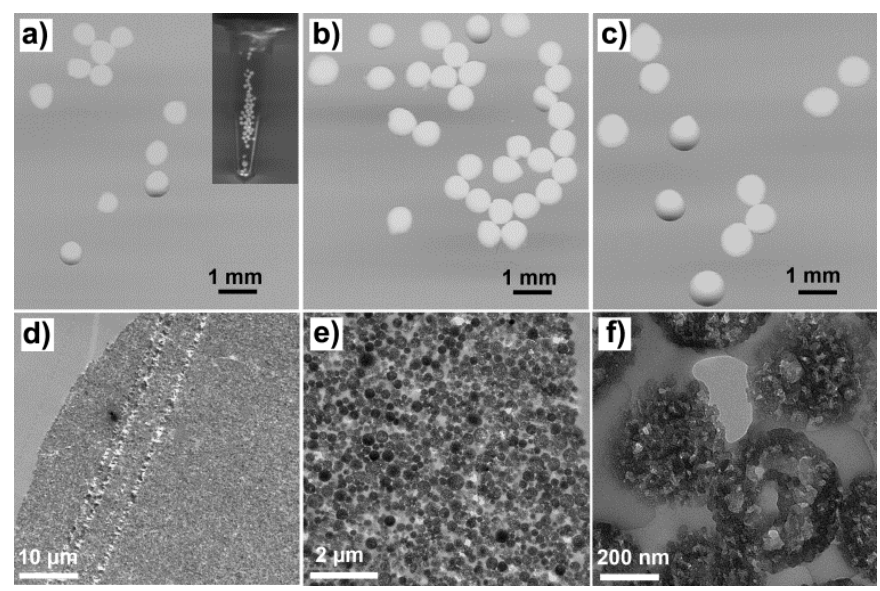

Figure 1. Digital photos of MS-SPs assembled from $0.6 \times 10^{8}$ (a), $1.2 \times 10^{8}$ (b) and $2.4 \times 10^{8}$ (c) MS nanoparticles. The inset in 1a is a photo of the MS-SPs collected in a $1 \mathrm{~mL}$ centrifuge tube. TEM images of ultramicrotomed MS-SPs as shown in (a) at different magnifications (df). 


\section{WILEY-VCH}
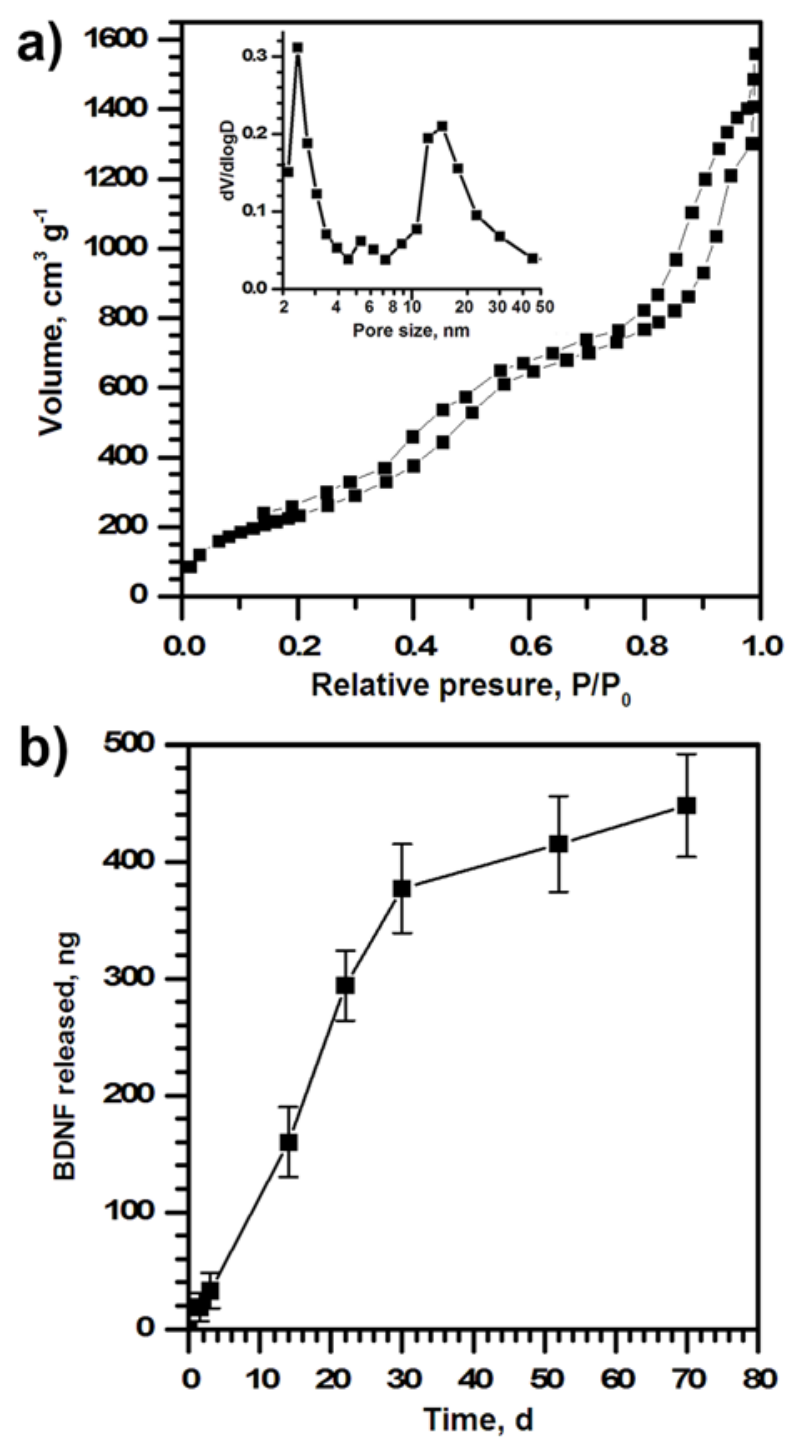

Figure 2. Nitrogen adsorption-desorption isotherms of MS-SPs (a) and in vitro release profile of BDNF-loaded MS-SPs (b). Inset of (a) is the pore size distribution of MS-SPs. A BDNF Emax ImmunoAssay (Promega) was used for the measurement of BDNF. 


\section{WILEY-VCH}
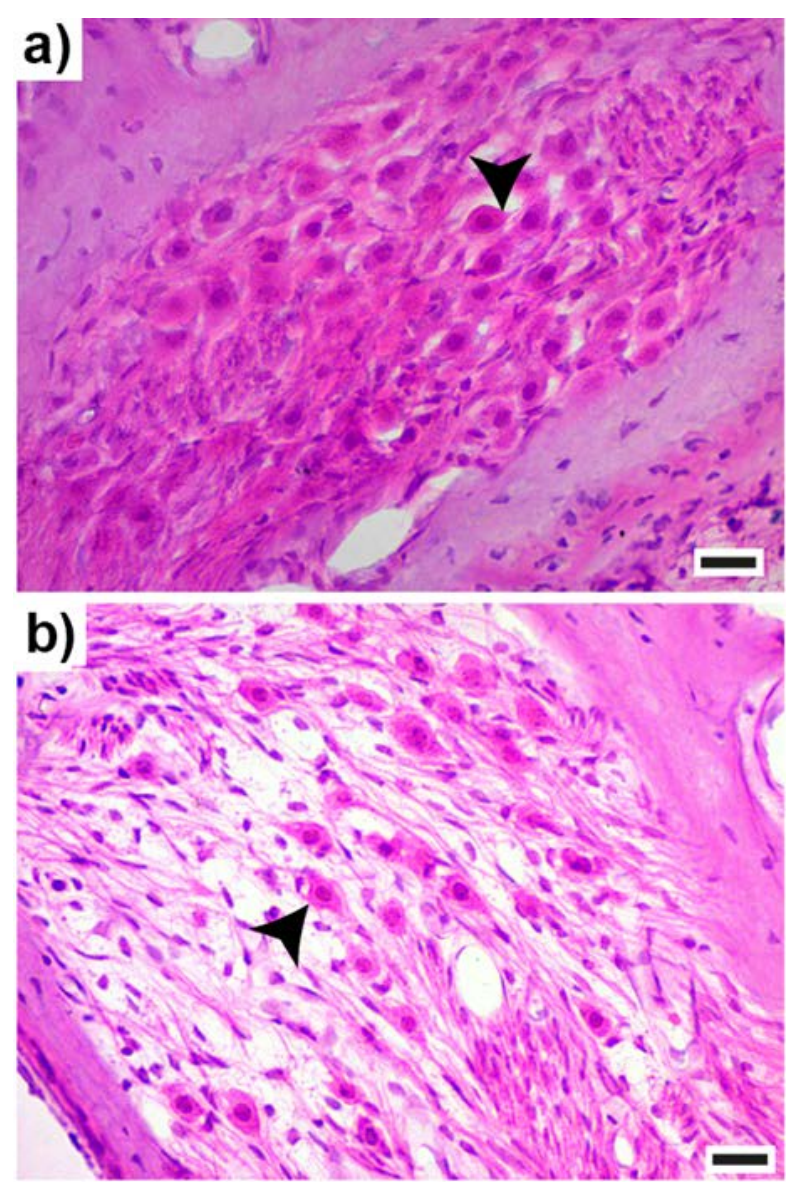

Figure 3. Histological images of a cochlea from a profoundly deafened guinea pig treated with BDNF-loaded (a) and unloaded (b) MS-SPs. The arrowheads indicate surviving ANs. Scale bars are $20 \mu \mathrm{m}$. 


\section{WILEY-VCH}

\section{Table of Content (TOC)}

Mesoporous silica supraparticles (MS-SPs) are prepared via self-assembly of mesoporous silica nanoparticles under capillary force action in confined droplets. The MS-SPs are effective carriers for sustained drug delivery. Animal study showed that these particles are suitable for chronic intracochlear implantation and neurotrophins released from the MS-SPs can efficiently rescue primary auditory neurons in an in vivo sensorineural hearing loss model.

\section{Keyword}

mesoporous materials, supraparticles, drug delivery, neurotrophic factors, sensorineural hearing loss

Yajun Wang, Andrew K. Wise, Justin Tan, James W. Maina, Robert K. Shepherd, and Frank Caruso*

Mesoporous Silica Supraparticles for Sustained Inner-Ear Drug Delivery

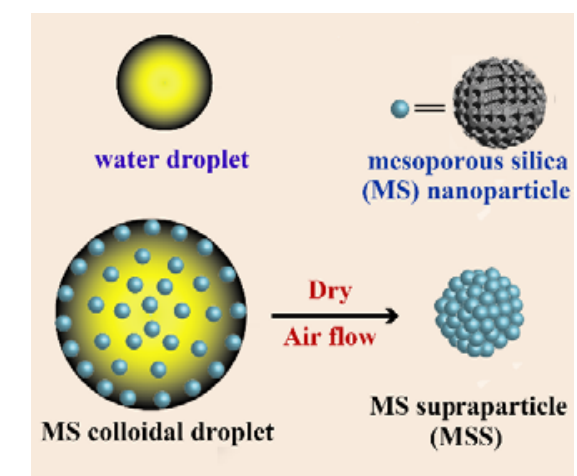




\section{University Library}

\section{- M M N E R VA A gateway to Melbourne's research publications}

Minerva Access is the Institutional Repository of The University of Melbourne

Author/s:

Wang, Y;Maina, JW;Caruso, F;Wise, AK;Shepherd, RK;Tan, J

Title:

Mesoporous Silica Supraparticles for Sustained Inner-Ear Drug Delivery

Date:

2014

Citation:

Wang, Y., Maina, J. W., Caruso, F., Wise, A. K., Shepherd, R. K. \& Tan, J. (2014).

Mesoporous Silica Supraparticles for Sustained Inner-Ear Drug Delivery. Small, 10 (21), pp.4244-4248. https://doi.org/10.1002/smll.201401767.

Persistent Link:

http://hdl.handle.net/11343/123272 